\title{
A Rare Skull Base Metastatic Follicular Thyroid Carcinoma Presenting as Sin nasal Carcinoma.
}

\author{
Rayapa Reddy Thumma ${ }^{1}$, Premalatha Pidakala ${ }^{2}$, Ravi Krishana ${ }^{3}$, \\ Vaddati Tejaswini ${ }^{4}$ \\ ${ }^{I}$ Department of Pathology, NRI Medical College, Dr. NTR University of Health Sciences, India \\ ${ }^{2}$ Department of Pathology, NRI Medical College, Dr. NTR University of Health Sciences, India \\ ${ }^{3}$ Department of Pathology, NRI Medical College, Dr. NTR University of Health Sciences, India \\ ${ }^{4}$ Department of Pathology, NRI Medical College, Dr. NTR University of Health Sciences, India
}

\begin{abstract}
Tumors that metastasize to paranasal sinus (PNS) and skull base are rare, with fewer than 200 cases reported worldwide. Of these, thyroid malignancies contribute $8 \%$. We discuss here a 51 year old female with multiple PNS metastases from follicular carcinoma of thyroid who had undergone right hemithyroidectomy 10 years back. She presented with nasal obstruction, progressive loss of vision and epistaxis 2 months. CECT scan of PNS showed a large heterogeneously enhancing mass lesion in the left nasal cavity crossing midline extending to right side and inferiorly into sphenoid and cavernous sinuses, which on further evaluation was found to be metastases from follicular carcinoma thyroid. To conclude PNS metastases from thyroid cancer are rare. But it should be always kept in mind in symptomatic patients.
\end{abstract}

Key words: Carcinoma thyroid, paranasal sinus metastasis, orbital metastasis.

\section{Introduction:}

Thyroid malignancies are among the frequently encountered head and neck malignancies. Follicular carcinoma of the thyroid arises from the follicular cells of the thyroid. Among the differentiated thyroid cancers $13-18 \%$ is follicular carcinoma [1]. They either occur de-novo or as a malignant transformation in a long standing thyroid swelling [2]. They usually present as a solitary nodule and sometimes with symptoms or signs of metastasis. Follicular carcinomas tend to invade locally and metastasize distantly to lungs and bones rather than to local nodes [3]. Till date only 22 cases of thyroid malignancy with metastasis to skull base have been reported.

\section{Case Report:}

A 51 year old female presented with progressive loss of loss of vision for 2 months, which was gradually increasing leading to complete loss of vision, epistaxis 1 month, headache associated with projectile vomiting since 2 weeks. She reported having right hemithyroidectomy 10 years back possibly for solitary nodule of thyroid but the details were not available. There was no history of trauma to the nose and no history of seizures

On clinical examination, nasal frame work appeared to be normal. Anterior rhinoscopy revealed deviated nasal septum to left with spur and on medializing the uncinate a soft pinkish friable mass seen behind the uncinate. Ocular examination showed protrusion of the eyeball with absence of pupillary reaction and no perception of light.

Ultrasound examination of neck showed post operative status of right lobe of thyroid and left lobe of thyroid measuring $3.4 \mathrm{X} 1.4 \times 1.6 \mathrm{~cm}$ with multiple iso to hypoechoic nodules with peripheral vascularity, largest measuring $1.4 \times 1.1 \mathrm{~cm}$. No calcifications seen. The thyroid profile was normal.

A contrast enhanced computer tomography (CECT) of PNS showed a well defined, lobulated intensely enhancing lesion measuring $5 \times 5.1 \times 5.4 \mathrm{~cm}$ in the left nasal cavity crossing midline and extending into right nasal cavity causing destruction of left lamina papiracyea, compression of medial rectus muscle and optic nerve causing proptosis of left eye. The lesion is extending inferiorly into sphenoid sinus and also to cavernous sinus with encashment of cavernous part of left internal carotid artery [Fig 1].

Excision of orbitoethmoidal tumor with completion thyroidectomy and orbital exenteration was done and subjected to histopathology.

2.1. Gross: Received left hemithyroidectomy specimen measuring $6 \mathrm{X} 4 \times 2 \mathrm{~cm}$. Cut section shows multiple circumscribed solid grey white nodules of varying sizes, largest measuring $1.3 \mathrm{~cm}$. Also received multiple grey 
brown tissue bits altogether measuring $5 \times 5 \mathrm{~cm}$ along with enucleation of left eye ball with intact optic nerve of $0.5 \mathrm{~cm}$ length [Fig 2].

2.2. Microscopy: Multiple sections studied from the thyroid showed nodules of varying sizes composed of micro and macro follicles filled with colloid, areas of haemorrhages, cystic change and fibrosis which were suggestive of nodular goitre [Fig 5]. Sections studied from the multiple bits show metastatic follicular carcinoma thyroid and paranasal sinus lining epithelium along with evidence of bone destruction

[Fig $3 \& 4$ ]. Sections studied from the optic nerve and eye ball are free from tumor deposit [Fig 6].

\section{Discussion:}

carcinoma is the second most common thyroid cancer, accounting for 15-20\% of all thyroid gland malignancies. Distant metastasis, although relatively uncommon, has been known to occur more commonly to the lungs and bone. Metastasis to paranasal sinuses and skull base is a rarely occurring phenomenon in thyroid malignancies. Skull metastasis is uncommon, and is found in $2.5 \%-5.8 \%$ of cases of thyroid cancer [4].

The method of spread of Thyroid Carcinoma into the skull and skull base is likely via the haematogenous route. Batson demonstrated a vertebral venous plexus which consisted of a valveless vascular bed within the spinal canal and extended from the skull to the pelvis [5]. Batson and Eckenhoff showed that there were multiple anastomoses and free connections between this venous plexus and the dural sinuses, the emissary veins of the skull as well as numerous junctions with the cervical plexus [6]. Recently arterial spread has also been suggested because of the association with secondary cutaneous locations in the territory of ipsilateral external carotid artery [7].

Matsuno et al [8] noted that the mean duration between initial diagnosis and metastasis to the skull base was 4 years. This cannot be applied to our patient because the exact duration of malignancy prior to metastasis is not known as the patient sought medical help only after the onset of epistaxis, which was nearly 10 years after previous right hemithyroidectomy.

Metastases to PNS are fairly uncommon, with a literature review from Germany listing 169 cases in 123 reports. Among these, thyroid gland was reported as the 4th commonest site of origin, this particular report listing 13 such cases [9]. The most commonly affected PNS was

maxillary sinus followed by sphenoid. Altman described 11 cases of metastatic thyroid cancers to PNS in a literature review [10]. Epistaxis was the most common symptom which was attributed to the hypervascular nature of thyroid tumor metastasizing to PNS.[11] Berstein, et al. described the review of 82 cases of PNS metastases. Forty patients had metastases in maxilla, 15 in ethmoid, 12 in frontal and six in sphenoid sinus. In $55 \%$ patients, primary site was identified as kidney. Thyroid primaries contributed only three cases [12].

Barrs et al. reviewed a series of eight patients with PNS metastases, two from prostate, two from myeloma, one from each kidney, lung, breast and follicular carcinoma thyroid [13].

In spite of extensive skull base metastasis our patient was in good general condition and was ambulatory. There is no evidence based treatment protocol for skull base metastasis in thyroid malignancy because of the rarity of the case. However, it is suggested that thyroidectomy and removal of as many metastasis as possible should be the mainstay of treatment. In skull base metastasis, this may not be feasible because of the presence of vital structures and possible profuse bleeding.

\section{Conclusion:}

This is a case of follicular carcinoma of the thyroid with rare site of metastasis to the skull base, presenting primarily with epistaxis, proptosis and right lateral rectus palsy. This not only stresses on the possibility of thyroid carcinoma metastasis to the skull base but also to evaluate thyroid malignancies with cranial nerve palsies for possible skull base metastasis.

\section{References:}

[1]. Cohen EG et al (2003) Postoperative management of differentiated thyroid cancer. Otolaryngol Clin N Am 36:129-157.

[2]. Ramsaden J, Watkinson JC (2008) Thyroid cancer. In: Glesson M (ed) Scott Brown's otorhinolaryngology and head and neck surgery, 7 th edn. 2663-2701.

[3]. İzci (2005) Brain metastasis from follicular thyroid carcinoma.Turk Neurosurg 15(3):132-135.

[4]. YAN Bo et al (2010) Papillary thyroid micro carcinoma presenting as skull base metastasis. Chin Med J 123(19):2750-2752.

[5]. Baston OV (1940) The function of the vertebral vein and their role in the spread of metastasis. Ann Surg 112:138-149.

[6]. Florence-Laigle D et al (2005) Skull-base metastases. J Neuro Oncol 75:63-66

[7]. Ortiz LP et al (2009) Thyroid follicular carcinoma presenting as skull and dural metastasis mimicking a meningioma: a case report. J Neurooncol 95:281-284.

[8]. Matsuno A et al (2010) Skull base metastasis from follicular thyroid carcinoma- two case reports. Neurol Med Chir Tokyo 50:421425.

[9]. Prescher A, Brors D. Metastases to the paranasal sinuses: Case report and review of the literature. Laryngorhinootologie 2001;80:583-94. 
[10]. Altman KW, Mirza N, Philippe L. Metastatic follicular thyroid carcinoma to paranasal sinus, a case report and review. J Laryngol Otol 1997;111:647-51.

[11]. Yamasoba T, Kikuchi S, Sugasawa M, Higo R, Sasaki T. Occult follicular carcinoma metastasizing to the sinonasal tract. ORL J Otorhinolaryngol Relat Spec 1994;56:239-43.

[12]. Bernstein JM, Montgomery WW, Balogh K. Metastatic tumors of the maxilla, nose, and paranasal sinuses. Laryngoscope 1966;76:621-50.

[13]. Barrs DM, McDonald TJ, Whisnant JP. Metastatic tumors to the sphenoid sinus. Laryngoscope 1979;89:1239-43.

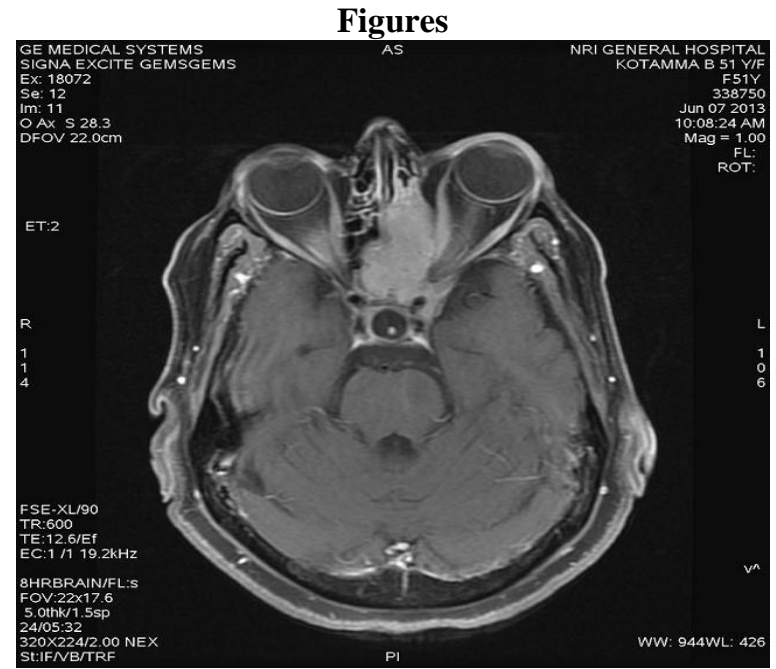

Fig-1: MRI showing intensely enhancing solid mass lesion at left orbital apex extending in to ethmoidal sinus and cavernous sinus with encasement of internal carotid artery

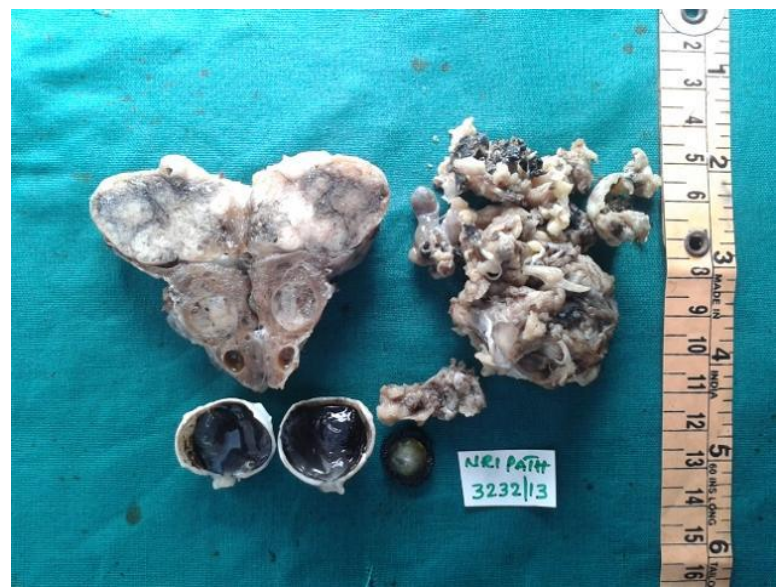

Fig-2: Cut section of Right hemithyroidectomy, eye ball with intact optic nerve and orbitoethmoidal tumor

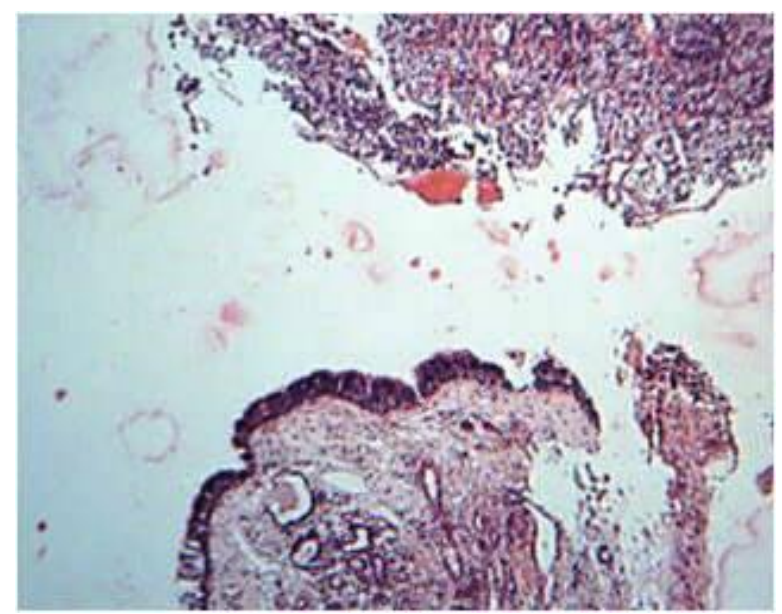

Fig-3: H\&E X40 showing tumor deposit along with adjacent bits showing sinonasal epithelium 


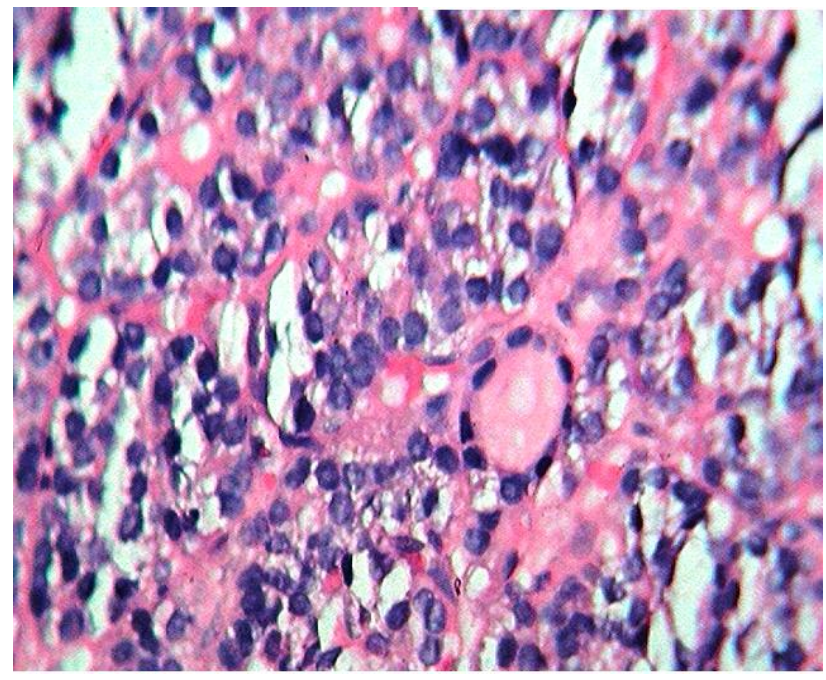

Fig-4: H\&E X100 showing metastatic deposit of follicular carcinoma thyroid, some of the follicles are filled with colloid.

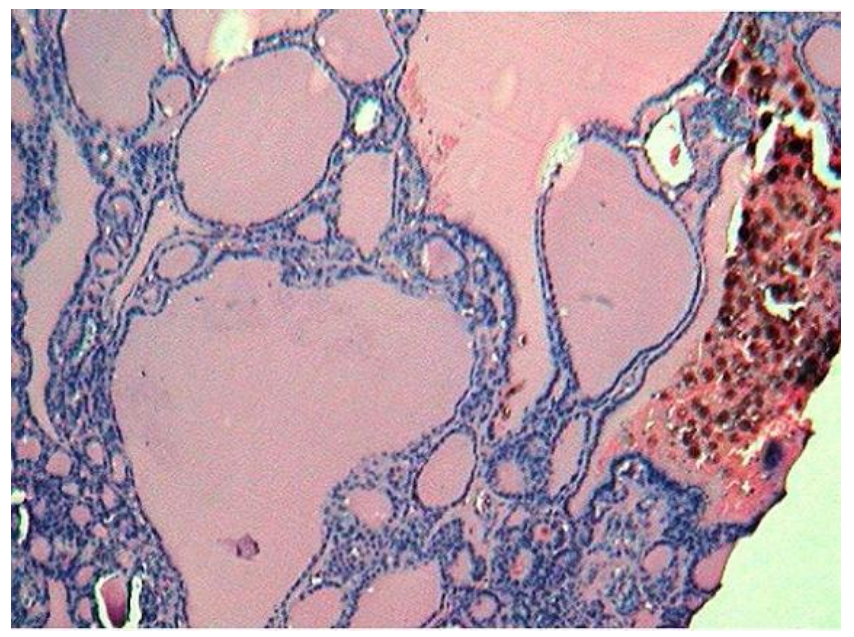

Fig-5: H\&E X100 showing thyroid follicles of varying sizes lined by flattened epithelium along with pigment laden macrophages

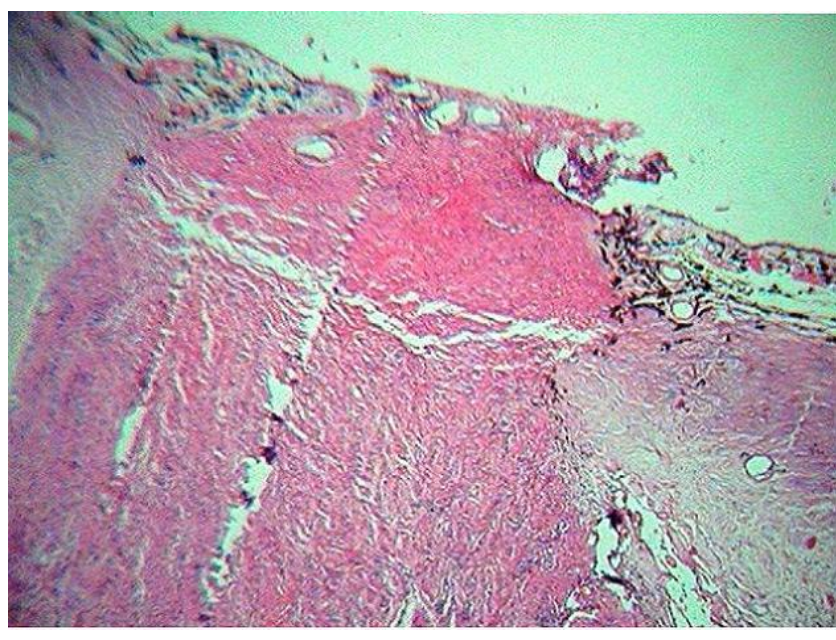

Fig-6: H\&E X100 of optic nerve and macula densa are free from tumor deposit 